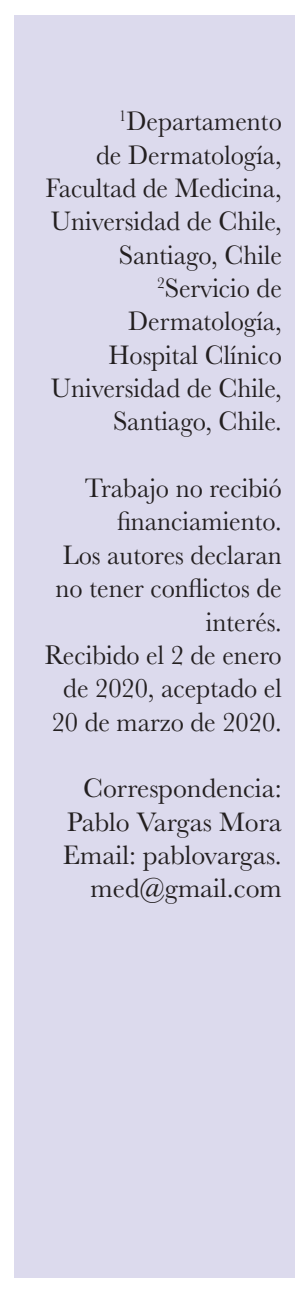

\title{
Gasos Guínicos
}

\section{Sebaceoma: hallazgos dermatoscópicos a propósito de dos casos}

Pablo Vargas ${ }^{1}$, Diego Orlandi ${ }^{1}$, Paula Giacaman ${ }^{1}$, Andrés Figueroa $^{2}$

\section{Resumen}

El sebaceoma es una neoplasia sebácea benigna inusual, que inicialmente se denominaba epitelioma sebáceo, lo que generaba confusión respecto a su biología tumoral, dado que histopatológicamente no presenta diferenciación suficiente como el adenoma sebáceo y tampoco es tan indeferenciado como el carcinoma sebáceo. Su diagnóstico precoz tiene gran relevancia, dado que, junto con el adenoma sebáceo y el carcinoma sebáceo, tienen una asociación directa con el síndrome de Muirr-Torre y, por lo tanto, con el desarrollo de cáncer de colon, endometrio, entre otros. En este punto, el uso de la dermatoscopia es muy importante.

Considerando los pocos reportes en la literatura sobre la dermatoscopia en sebaceomas, presentamos dos casos clínicos en adultos, donde se destaca la presencia de estructuras amarillas homogéneas y vasos en corona o arboriformes.

Palabras claves: Sebaceoma; dermatoscopía; síndrome de Muir-Torre.

\section{SUMMARY}

Sebaceoma is an unusual benign sebaceous neoplasm, initially known as sebaceous epithelioma, which generated confusion regarding its tumor biology, given that it is histopathologically less differentiated than sebaceous adenoma, but with a higher differentiation than sebaceous carcinoma. Early diagnosis becomes relevant, given that together with sebaceous adenoma and sebaceous carcinoma, there is a direct association with Muirr-Torre syndrome and therefore the development of colon and endometrial cancer, among others. Because of this, the use of dermatoscopy becomes very important. Given the few reports in the literature on dermatoscopy in sebaceomas, we present two clinical cases, where the presence of homogeneous yellow structures and crown or arboriform vessels stands out.

Key words: Sebaceoma; dermatoscopy; MuirTorre syndrome.
$\mathrm{E}$ 1 sebaceoma es una neoplasia sebácea benigna inusual, que previo a su primera descripción por Troy y Ackerman en $1984^{1}$, se denominaba epitelioma sebáceo, generando confusión respecto a su biología tumoral, dado que histopatológicamente no presenta diferenciación suficiente como para llamarlo adenoma sebáceo y tampoco es tan indeferenciado como el carcinoma sebáceo o carcinoma basocelular con diferenciación sebácea ${ }^{2}$. Al igual que otros tumores sebáceos, exceptuando la hiperplasia sebácea, se relaciona al Síndrome de Muir-Torre, por lo que su diagnóstico precoz y estudio tienen gran relevancia ${ }^{2,3}$. Considerando la indudable utilidad de la dermatoscopía como herramienta diagnóstica y dado los escasos reportes existentes en la literatura sobre su aplicación en sebaceomas, se presentan dos casos clínico-dermatoscópicos.

\section{Casos clínicos}

Una mujer de 50 años, sana. Presenta hace un año una lesión asintomática en el ala nasal izquierda, de crecimiento lentamente progresivo. Al examen físico se observa una pápula ovalada, sésil, amarillenta, con superficie lisa y bordes regulares, de $3 \mathrm{~mm}$. de diámetro mayor (Figura 1A). A la dermatoscopía destaca una lesión rosado pálida, con áreas amarillentas centrales y vasos arboriformes aislados en la periferia (Figura 1B). Se realiza biopsia excisional, que muestra una proliferación epitelial dérmica, nodular, con lóbulos de células de núcleos pequeños, ovoides, de cromatina fina, con escaso citoplasma, de aspecto basaloide, sin evidente actividad mitótica, entremezclados con escasos sebocitos normotípicos. 

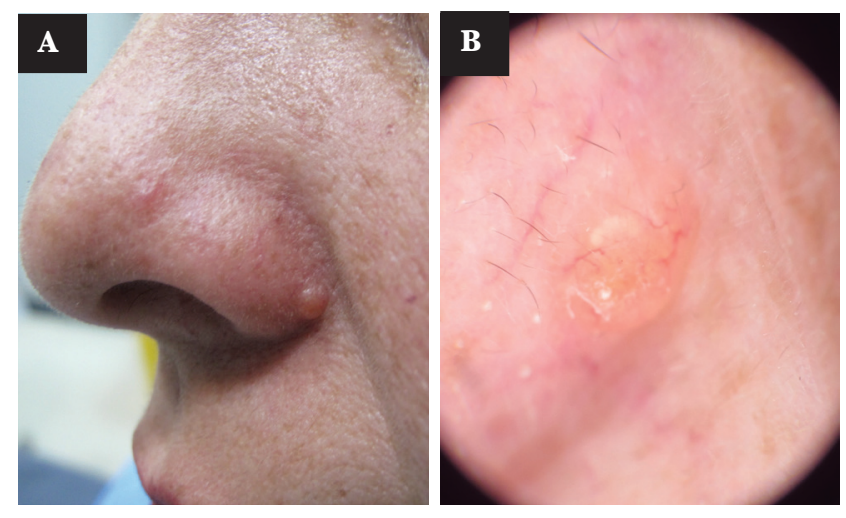

\section{Figura 1}

A. Pápula sésil, amarillenta, de superficie lisa en el ala nasal izquierda.

B. Dermatoscopía: lesión rosado pálida con centro amarillento y vasos arboriformes desenfocados en periferia.

El segundo caso corresponde a un hombre de 53 años, sano. Consulta por una pápula asintomática de 8 meses de evolución, en el párpado inferior izquierdo. A la exploración física se observa una pápula cupuliforme levemente eritematosa, de $5 \mathrm{~mm}$ de diámetro mayor, bordes regulares y superficie lisa, con algunas telangiectasias. En la evaluación dermatoscópica, se aprecia una lesión rosada homogénea con un área central amarillenta-blanquecina y vasos finos lineales y serpinginosos periféricos, distribuidos en corona (Figura 2A). El estudio histopatológico revela una proliferación epitelial dérmica simétrica, con células basaloides entremezcladas con algunos sebocitos maduros y conductos sebáceos (Figura 2B).
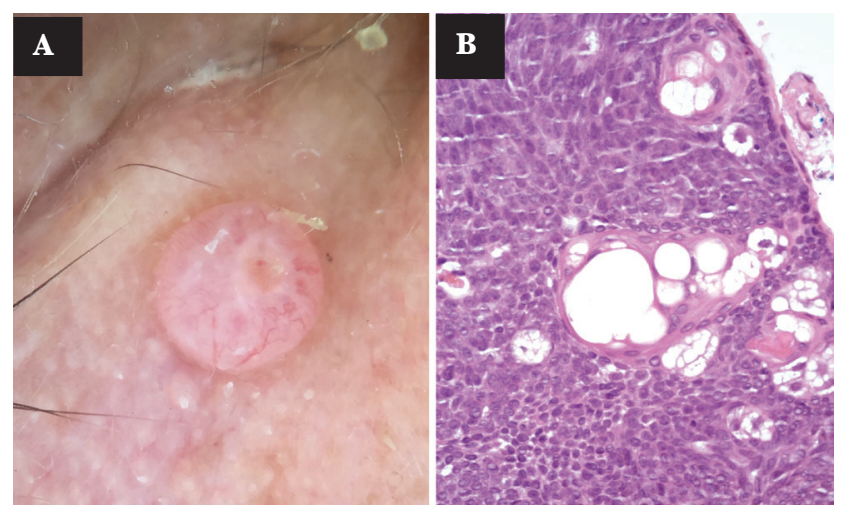

\section{Figura 2}

A. Dermatoscopía: lesión rosada con área central amarillentablanquecina, vasos finos periféricos distribuidos en corona.

B. Histopatología: proliferación epitelial dérmica simétrica, con células basaloides entremezcladas con algunos sebocitos maduros y conductos sebáceos (100x, H/E).

\section{Discusión}

El sebaceoma clínicamente se manifiesta como una pápula o nódulo de hasta $2 \mathrm{~cm}$, de superficie lisa, color amarillo, de crecimiento lento y ubicado más frecuentemente en cabeza y cuello ${ }^{2,4}$. Histopatológicamente se encuentra compuesto por múltiples nidos de células basaloides, que corresponden a sebocitos inmaduros monomorfos y que según la definición clásica deben representar más del 50\% de las células tumorales, lo que hace la diferencia versus el adenoma sebáceo ${ }^{4}$. Para algunos autores, ambas lesiones representarían distintos grados de maduración de una misma neoplasia basaloide benigna con diferenciación sebácea, denominándola

Tabla 1

Hallazgos dermatoscópicos en Sebaceomas.

\begin{tabular}{|c|c|c|c|c|c|c|c|c|}
\hline $\mathbf{N}^{0}$ & Ref. & Ubicación & $\begin{array}{c}\text { Áreas } \\
\text { blanquecinas }\end{array}$ & $\begin{array}{c}\text { Áreas } \\
\text { amarillentas }\end{array}$ & $\begin{array}{c}\text { Vasos } \\
\text { en corona }\end{array}$ & $\begin{array}{c}\text { Vasos } \\
\text { arboriformes }\end{array}$ & Ulceración & $\begin{array}{c}\text { Áreas } \\
\text { rosadas }\end{array}$ \\
\hline 1 & (7) & Cuero cabelludo & + & + & + & - & - & - \\
\hline 2 & (6) & Cara & + & + & + & - & + & - \\
\hline 3 & (6) & Torso & + & + & + & - & - & - \\
\hline 4 & (8) & $\mathbf{N} / \mathbf{R}$ & - & + & + & - & - & - \\
\hline 5 & (8) & $\mathbf{N} / \mathbf{R}$ & - & + & + & - & - & - \\
\hline 6 & (9) & Sacro & - & + & - & + & + & + \\
\hline 7 & (10) & Mama & - & + & - & - & - & - \\
\hline 8 & Caso 1 & Nariz & - & + & - & + & - & + \\
\hline 9 & Caso 2 & Párpado & + & + & + & - & - & + \\
\hline
\end{tabular}

$\mathcal{N} / R$ : no registrado 
sebomatricoma ${ }^{5}$. Las células basaloides pueden formar diversos patrones de crecimiento, tales como: laberíntico-sinusoidal, carcinoide-like u ondulado.

Su diagnóstico clínico precoz cobra relevancia, dado que, junto al adenoma sebáceo y carcinoma sebáceo, existe una asociación directa con el Síndrome de MuirTorre y, por ende, el desarrollo de cáncer de colon, endometrio, gástrico, entre otros, lo que justifica el estudio oportuno de neoplasia visceral ${ }^{3,5}$. Por esto, es de gran importancia el uso de la dermatoscopía, de la cual existen sólo 7 casos reportados en la literatura, describiendo con más frecuencia áreas amarillentas y vasos, los que pueden ser arboriformes o distribuirse en corona, tal como los dos casos del presente reporte, siendo menos habitual el hallazgo de áreas blanquecinas, fondo rosado o ulceración ${ }^{6-10}$ (Tabla 1). Si bien el diagnóstico diferencial clínico y dermatoscópico con hiperplasia sebácea suele ser sencillo, esto no es así con tumores como el adenoma sebáceo o el carcinoma sebáceo, aun cuando de este último se ha descrito en pequeñas series el predominio de vasos polimorfos en un fondo amarillo ${ }^{8}$.

\section{Conclusión}

Considerando los múltiples patrones histopatológicos descritos en el sebaceoma, junto a la dificultad en el diagnóstico clínico y a menudo microscópico con otras tumoraciones sebáceas ${ }^{8}$, se hace necesario el diseño de estudios con un mayor número de casos, para evaluar si existen diferencias dermatoscópicas claras entre ellos, de tal forma de eventualmente prescindir de la biopsia y pesquisar precozmente neoplasias viscerales, en contexto de Síndrome de Muir-Torre.

\section{REFERENCIAS}

1. Troy JL, Ackerman AB. Sebaceoma. A distinctive benign neo-plasm of adnexal epithelium differentiating toward sebaceouscells. Am J Dermatopathol 1984; 6: 7-13

2. Bourlond F, Velter C, Cribier B. Clinicopathological study of 47 cases of sebaceoma. Ann Dermatol Venereol. 2016; 143: 814-824.

3. Iacobelli J, Harvey NT, Wood BA. Sebaceous lesions of the skin. Pathology. 2017; 49: 688-697

4. Flux K. Sebaceous Neoplasms. Surg Pathol Clin. 2017Jun;10(2):367382.

5. Sánchez Yus E, Requena L, Simon P, del Rio E. Sebomatricoma: a unifying term that encompasses all benign neoplasms with sebaceous differentiation. Am J Dermatopathol 1995; 17: 213-21

6. Moscarella E, Argenziano G, Longo C, Cota C, Ardigò M, Stigliano $\mathrm{V}$, et al. Clinical, dermoscopic and reflectance confocal microscopy features of sebaceous neoplasms in Muir-Torre syndrome. J Eur Acad Dermatol Venereol. 2013; 27: 699-705

7. Nomura M, Tanaka M, Nunomura M, Izumi M, Oryu F. Dermoscopy of Rippled Pattern Sebaceoma. Dermatol Res Pract. 2010; 2010. pii: 140486

8. Lallas A, Moscarella E, Argenziano G, Longo C, Apalla Z, Ferrara G, et al. Dermoscopy of uncommon skin tumours. Australas J Dermatol. 2014; 55: 53-62

9. Coppola R, Carbotti M, Zanframundo S, Rinati MV, Graziano A, Panasiti V. Use of dermoscopy in the diagnosis of sebaceoma. J Am Acad Dermatol. 2015; 72: e143-5

10. Fernandez-Flores A, Manjón JA. Sebaceoma associated with seborrheic keratosis. Rom J Morphol Embryol. 2017; 58: 599-601 